\title{
REFERENCES
}

1. M. W. Al-Dhahir and R. N. Shekoury, Constructions in the hyperbolic plane, Proc. Iraqi Sci. Soc. 2 (1958), 1-6.

2. H. S. M. Coxeter, Non-euclidean geometry, 3d ed., Univ. of Toronto Press, 1957.

3. F. Handest, Constructions in hyperbolic geometry, Canad. J. Math. 8 (1956), 389-394.

UNIVERSITY OF BAGHDAD AND

UNIVERSITY OF TORONTO

\section{ON THE EULER CHARACTERISTIC OF POLYHEDRA}

\section{CHARLES E. WATTS ${ }^{1}$}

1. The principal object of this note is to give an extremely simple axiomatic characterization of the Euler characteristic for finite polyhedra. The idea of the construction used was suggested by the definition of the Grothendieck group of sheaves over a space [1]. Unless otherwise noted, "space" always means "triangulable space with base point," and a "pair" is a "triangulable pair of spaces with common base point." Mappings of spaces always preserve base points. If $(X, A)$ is a pair, then $X / A$ denotes the space obtained from $X$ by identifying $A$ to a point.

2. Let $F$ be the free abelian group generated by all homeomorphism classes of spaces. We denote by $N$ the subgroup of $F$ generated by all elements of the form

$$
X-A-X / A \text {, }
$$

where $(X, A)$ is a pair. Set $\Gamma=F / N$ and let $\gamma: F \rightarrow \Gamma$ be the natural homomorphism.

Lemma 1. If $X, Y$ are spaces, then $\gamma(X \vee Y)=\gamma X+\gamma Y$.

The proof is obvious.

Lemma 2. If $E^{n}$ is an $n$-cell (with base point on the boundary) then $\gamma E^{n}=0$.

Received by the editors February 27, 1961.

1 This work was done while the author was a National Science Foundation Postdoctoral Fellow. 
Proof. Consider the pair $\left(E^{n+1}, E^{n}\right)$ where $E^{n}$ is imbedded as a boundary cell. The identification space being an $E^{n+1}$, we have $\gamma E^{n+1}=\gamma E^{n}+\gamma E^{n+1}$, whence the result.

Lemma 3. If $S^{r}$ denotes the $r$-sphere, then $\gamma S^{n}=-\gamma S^{n+1}$, $r=0,1,2, \cdots$.

Proof. Consider the pair $\left(E^{n+1}, S^{n}\right)$ where $S^{n}$ is imbedded as the boundary of $E^{n+1}$. The identification space being an $S^{n+1}$, we have $\gamma E^{n+1}=0=\gamma S^{n}+\gamma S^{n+1}$.

Lemma 4. $\Gamma$ is generated by $\gamma S^{0}$.

Proof. Let $X$ be any space, and let $K$ be a simplicial complex with $|K|$ homeomorphic to $X$, the base point of $X$ corresponding to a vertex of $K$. If $\operatorname{dim} K=0$, then $X$ is a wedge of 0 -spheres, and Lemma 1 implies that $\gamma X$ is an integral multiple of $\gamma S^{0}$. Proceeding by induction, suppose that $\gamma Y$ is an integral multiple of $\gamma S^{0}$ if $Y$ is homeomorphic to a complex of dimension $<n$, and let $X, K$ be as above with $\operatorname{dim} K=n$. Let $L$ be the $(n-1)$-skeleton of $K$. Now $|K| /|L|$ is a wedge of spheres and we have

$$
\gamma X=\gamma L+\gamma(|K| /|L|),
$$

so Lemmas 1 and 3 can be applied to complete the proof.

Lemma 5. $\Gamma$ is an infinite cyclic group.

Proof. Consider the function defined on the natural basis for $F$ which assigns to each space its reduced Euler characteristic (i.e., the usual Euler characteristic minus one, or the alternating sum of the ranks of the reduced homology groups). This function extends uniquely to a homomorphism $F \rightarrow Z$, vanishing on $N$, hence induces a map $\Gamma \rightarrow Z$. Since this map assumes the value 1 on $\gamma S^{0}$ and since $\Gamma$ is cyclic by the preceding lemma, the result follows.

THEOREM. Let $\epsilon$ be any integer-valued function defined for triangulable spaces with base point, such that

(1) $\epsilon X=\epsilon A+\epsilon(X / A)$ for any pair $(X, A)$;

(2) $\epsilon S^{0}=1$.

Then $\epsilon$ is the reduced Euler characteristic.

Proof. Let $\chi$ be the function assigning to each space its reduced Euler characteristic; $\chi$ also satisfies (1) and (2). Because of (1), $\epsilon$ and $\chi$ induce maps $\bar{\epsilon}$ and $\bar{\chi}: \Gamma \rightarrow Z$. Because of (2), $\bar{\epsilon} \bar{\chi}^{-1}$ carries the integer 1 into itself, hence is the identity map. This proves $\bar{\epsilon}=\bar{\chi}$ and $\epsilon=\chi$. 
3. If $X, Y$ are spaces we define the smash product $X \# Y$ to be $(X \times Y) /(X \vee Y)$. Then the group $F$ above has a ring structure as the integral algebra of the monoid of all spaces under \#. It is easy to verify that $N$ is a two-sided ideal of $F$, so that $\Gamma$ inherits this ring structure. As a result, we get an easy proof of the fact that the Euler characteristic of the Cartesian product of spaces is the product of their Euler characteristics.

4. We conclude by suggesting two related problems. First, we remark that the group $\Gamma$ has been defined essentially by abstracting the definition of Grothendieck to a category by formally replacing "exact sequence of sheaves" by "simplicial cofibration." This suggests that we similarly replace it by "simplicial fibration"; i.e., define the subgroup $N^{\prime}$ of $F$ to be that generated by all elements of the form $E-X-Y$, where there exists a fibering of $E$ with fiber $Y$ and base space $X$, and then set $\Gamma^{\prime}=F / N^{\prime}$. The study of this group $\Gamma^{\prime}$ appears to be quite difficult, and we have as yet no explicit information about it.

The second problem is to define and study other topological invariants in analogous ways. For example, let $N^{\prime \prime}$ be the subgroup of the group $N$ above where we allow only those generators corresponding to pairs $(X, A)$ such that $A$ is a retract of $X$. Since the homology sequence of such a pair splits in each dimension with any coefficient group, we get in an evident way many nontrivial homomorphisms of $\Gamma^{\prime \prime}=F / N^{\prime \prime}$ into the integers by assigning to each space its $n$th Betti number $\bmod p, n$ and $p$ arbitrary. The question arises as to whether these "Betti number" homomorphisms determine $\Gamma^{\prime}$ completely, in the manner in which the "Euler number" homomorphism determines $\Gamma$. Again, we have no explicit information.

\section{REFERENCE}

1. A. Borel and J.-P. Serre, Le theoreme de Riemann-Roch, Bull. Soc. Math. France 86 (1958), 97-136.

INSTITUTE FOR ADVANCED STUDY 